are required to describe injury occurrence and inform injury prevention measures.

Objective To analyse injuries sustained by professional rugby union players in Scotland.

Design Prospective observational.

Setting Time-loss match injuries sustained in men's and women's international rugby, men's professional club rugby and men's and women's international sevens during the 2017/18 and 2018/19 seasons were recorded by Scottish Rugby medical staff. Match exposure was recorded by GPS device and/or video analysis.

Patients (or Participants) Across all cohorts, 208 players (men: 163; women: 45) participated during the 2017/18 and 2018/ 19 seasons (men's international $\mathrm{n}=60$; women's international $\mathrm{n}=37$; men's professional club $\mathrm{n}=134$; men's international sevens $\mathrm{n}=29$; women's international sevens $\mathrm{n}=25$ ). Several players represented multiple cohorts.

Interventions (or assessment of Risk Factors) Injuries within and between cohorts were compared.

Main Outcome Measures Injury incidence, severity, type and location.

Results Injury incidences were 292.8 (95\% CI: 227.8-358.0)/ 1000 player match hours for men's international sevens, 183.3 (139.5-227.1)/1000 hours for women's international rugby, 167.5 (81.1-254.1)/1000 hours for women's international sevens, 160.0 (124.1-195.9)/1000 hours for men's international rugby, and 154.5 (140.2-168.8)/1000 hours for men's professional club. Median severity ranged from 6.0 - 19.5 days. Concussion (men's international: 22.5/1000 hours; women's international: 26.7/1000 hours; men's professional club: 28.9/1000 hours; men's international sevens: 37.3/1000 hours) was the most common injury for all cohorts except women's international sevens, where knee sprain/ligament injury was most frequent (41.9/1000 hours).

Conclusions Men's international sevens had the greatest injury incidence. Concussion was the most frequent injury in all cohorts except women's international sevens, where it was the second most common. Interventions to reduce concussion incidence would benefit all professional cohorts in Scotland.

\section{UTILITY OF THE HEALTH ACTION PROCESS APPROACH (HAPA) MODEL TO PREDICT INTENTION AND ADOPTION OF THE ACTIVATE INJURY PREVENTION EXERCISE PROGRAMME BY SCHOOL RUGBY COACHES}

${ }^{1}$ Craig Barden, ${ }^{1,2}$ Carly McKay, ${ }^{1,3}$ Keith Stokes. ${ }^{1}$ Department for Health, University of Bath, Bath, BA2 7AY, UK., Bath, UK; ${ }^{2}$ Centre for Motivation and Health Behaviour Change, University of Bath, Bath, BA2 7AY, UK., Bath, UK; ${ }^{3}$ Rugby Football Union, Twickenham, TW2 7BA, UK, London, UK

\subsection{6/bjsports-2021-IOC.225}

Background Using Activate, a rugby-specific injury prevention exercise programme, has been shown to reduce injury risk in school rugby; however, implementing such programmes is challenging and adherence is often suboptimal. The Health Action Process Approach (HAPA) model is a promising theory for identifying modifiable determinants of programme uptake.

Objective To evaluate the utility of the HAPA model when predicting coaches' intentions to use Activate, and to explore the relationship between intention and programme adoption.

Design Prospective cohort study.

Setting English schools rugby (under-12 to under-18).

Participants Rugby coaches and support staff $(n=38)$.
Interventions All coaches attended a pre-season Activateworkshop. Coaches completed a questionnaire pre- and post-workshop, assessing HAPA predictors: risk perception, outcome expectancies, task self-efficacy, intention, action and coping planning, maintenance self-efficacy and recovery self-efficacy.

Main outcome measures Standardized regression coefficients $(\beta)$ were used to measure the association between HAPA variables. Goodness-of-fit was assessed using the Comparative Fit Index (CFI), Tucker Lewis Index (TLI) and root mean square error of approximation (RMSEA). Programme adoption was selfreported at post-season.

Results Coaches' outcome expectancies $(\beta=0.33, \mathrm{p}<0.05)$ and task self-efficacy $(\beta=0.40, \mathrm{p}<0.01)$ were significantly associated with intention to use Activate $\left(\mathrm{r}^{2}=0.28\right)$. The model demonstrated good fit to predict intention $(\mathrm{CFI}=0.95$, TLI $=1.00$, RMSEA $=0.00)$. Task self-efficacy $(\beta=0.49, \mathrm{p}<0.01)$ and inten$\operatorname{tion}(\beta=0.27, \mathrm{P}<0.05)$ were significantly associated with action and coping planning $\left(r^{2}=0.43\right)$, though the relationship between intention and adoption was not significant $(\beta=0.09$, $\mathrm{p}=0.63)$.

Conclusions As hypothesised by the HAPA model, outcome expectancies and task self-efficacy were significant predictors of intention, and behaviour change strategies should focus on improving these. Predicted pathways between task self-efficacy, intention, and planning were also supported, though the model was underpowered to assess relationships between post-intentional variables and programme adoption. The model's utility beyond intention needs further exploration with larger sample sizes to identify additional intervention targets.

\section{SAFE AND SOUND FOR PERFORMANCE'S SAKE? AN EXPLORATION ON HEALTH AND SAFETY AWARENESS IN ELITE RUGBY}

${ }^{1}$ Yanbing Chen, ${ }^{2}$ Conor Buggy, ${ }^{1}$ Seamus Kelly. ${ }^{1}$ Institute of Sport and Health, School of Public Health, Physiotherapy and Sports Science, University College Dublin, Dublin, Ireland; ${ }^{2}$ Centre for Safety and Health at Work, School of Public Health, Physiotherapy and Sports Science, University College Dublin, Dublin, Ireland

\subsection{6/bjsports-2021-IOC.226}

Background In elite rugby union, players often expose themselves to risk-taking behaviours resulting in a high risk acceptance level. The practical and theoretical occupational safety and health $(\mathrm{OSH})$ have the potential to reflect health outcomes (e.g., injuries and accidents) in sports settings.

Objective This study explores key indicators of injury prevention and welfare protection in rugby union from an $\mathrm{OSH}$ perspective.

Design This study utilises semi-structured interview, the duration of which ranged from 22 to 50 minutes digitally recorded with consents.

Setting Individual interviews were conducted with current rugby supporting staff involving in national, provincial and university level.

Patients (or Participants) The participants $(n=15)$ were current rugby supporting staff including coaching staff, medical staff and other management personnel.

Interventions (or Assessment of Risk Factors) The interview transcripts were inductively analysed by using Nvivo software, the key risk factors were then identified using abductive analysis by adopting an existing safety climate framework. 
Main Outcome Measurements Factors being neglected in sports research will be discussed in this study, for example, safety justice is relating to players' risk-taking behaviours during match or training; whether opponents are 'co-workers' and players' safety attitudes towards co-workers can influence players' aggressiveness which relates to injuries and accidents.

Results The framework identified for evaluating OSH awareness will be presented from two dimensions including five themes: rugby management commitment (management safety priority, management safety empowerment and management safety justice) and rugby player involvement (players' safety priority and players' trust in co-workers' safety competence).

Conclusions The findings have theoretical implications for rugby organisations to design a survey to facilitate the development of appropriate behaviour interventions. Furthermore, the framework could be potentially applied in wider sports settings.

\section{ABSTRACT WITHDRAWN}

\section{U.S. RUGBY-7S PLAYERS INJURY INCIDENCE, SEVERITY AND BURDEN EFFECTS BY POSITIONS AND LEVELS OF PLAY}

1,2,3,4Victor Lopez, 1,2,5 Shen-Ying Richard Ma, 1,6 Meryle G Weinstein, 2Patria Hume $7,8,9,10,11$ Robert C Cantu, ${ }^{1,12}$ Christian Victoria, ${ }^{1}$ Sophie C Queler, ${ }^{1,13}$ Khalil JA Webb, 1,2,14,15,16 Answorth A Allen. 'Rugby Research and Injury Prevention Group, affiliate of Hospital for Special Surgeny, New York, USA; ${ }^{2}$ Sports Performance Research Institute New Zealand, Auckland University of Technology, Auckland, New Zealand; ${ }^{3}$ USA Rugby Empire and New England Geographic Union Rugby Football Unions, New York/Boston, USA; ${ }^{4}$ Northeast Rugby Academy, USA Rugby Development Program and USOC-Community Olympic Development Program, New York/Boston, USA; ${ }^{5}$ University of Missouri, Missouri Orthopaedic Institute and Thompson Laboratory for Regenerative Orthopaedics, Columbia, USA; ${ }^{6}$ New York University, Steinhardt School of Culture, Education and Human Development, New York, USA; ${ }^{7}$ Center for the Study of Traumatic Encephalopathy, Boston University School of Medicine, Boston, USA; ${ }^{8}$ Department of Neurosurgery and Sports Medicine, Emerson Hospital, Concord, USA; ${ }^{9}$ Neurologic Sports Injury Center, Brigham and Women's Hospital, Boston, USA; ${ }^{10}$ Concussion Legacy Foundation, Waltham, USA; ${ }^{11}$ World Rugby, Independent Concussion Group, Dublin, Ireland; ${ }^{2}$ New York University, College of Global Public Health, Urban Epidemiology Lab, New York, USA; ${ }^{13}$ University of Arizona, Tucson, USA; ${ }^{14}$ Sports Medicine and Shoulder Service, Hospital for Special Surgery, New York, USA; ${ }^{15}$ National Basketball Association, New York Knickerbockers, New York, USA; ${ }^{16}$ USA Basketball, Colorado Springs, USA

\subsection{6/bjsports-2021-IOC.227}

Background There are limited injury data for Rugby-7s, and even less data analysed by participation level or days returnto-sport after injury.

Objective To describe injury incidence, severity, and injury burden for three levels of Rugby-7s competition.

Design Prospective descriptive epidemiology study.

Setting U.S. Rugby-7s tournaments/series and championships $(\mathrm{n}=57$; 2010-2014) over 72 tournament days; L1 elite, L2 sub elite, and L3 under-19/college/senior games (exposure $=14,591$ player-hours).

Participants 24,538 U.S. players $(\mathrm{men}=17,770$; women=6,768; age $13-54$ years).

Assessment of Risk Factors Intrinsic and extrinsic risk in match injuries.

Main measurement outcome Incidence (per/1000 player-hour (ph)) and mechanism of injury were captured using Rugby
Injury Survey \& Evaluation (RISE) report methodology. Timeloss injuries, injury severity $($ days $=\mathrm{d})$ from training/competition (including post tournament) were documented, and injury burden were calculated.

Results Injury incidence $(n=491)$ was not significantly different between levels (L3:30.74/1000ph, CI:27.26-34.54; L2:36.24/1000ph, CI:30.84-42.31; L1:41.78/1000ph, CI:30.8-55.39). Mean injury burden was significantly lower for L3 than L1 and L2 (L3-vs-L2, $P<0.001$; L3 vs L1, $P<0.001)$. Greater risk of lower limb injuries was noted in L3-vs-L1 (RR:0.59, CI:0.38-0.95, $P=0.024$ ). The cohort sustained high head/neck injury rates $(22.6 \% ; 13.3 / 1000 \mathrm{ph})$. Backs had more injuries among levels than forwards (L1 backs 51.8/1000ph, forwards $26.4 / 1000 \mathrm{ph}, P=0.034$; L2 backs $37.7 / 1000 \mathrm{ph}$, forwards $29.6 / 1000 \mathrm{ph}, P=0.152 ; \mathrm{L} 3$, backs $32.76 / 1000 \mathrm{ph}$, forwards $24.8 / 1000 \mathrm{ph}, P=0.029$; total cohort backs 35.74/1000ph, forwards 26.39/1000ph, RR:1.35; CI:1.12-1.65, $P=0.002)$. Average days absent post injury $=44.0 \mathrm{~d}(37.8-50.1 \mathrm{~d})$ in $68.4 \%$ with follow-up data. A significant difference $(P=0.018)$ in mean severity days absent from sport was between the L3 (57.1d) and L2 (27.9d) forwards.

Conclusions Competition level and playing position had significant effects on injury burden and nature of injury. The L1 and L2 had higher injury burden than the lower L3. The L1vs-L3 cohort had a high proportion of head/neck injury risk compared with other injury locations. Backs sustained greater injury incidence rates among all three levels as compared to forwards.

\section{THE EPIDEMIOLOGY OF HEAD, NECK AND FACE INJURIES OF ADULT MEN'S AND WOMEN'S U.S. RUGBY-7S PLAYERS}

${ }^{1,2,3}$ Shen-Ying Richard Ma, ${ }^{2,4}$ Christian Victoria, ${ }^{4}$ Danielle C Ompad, ${ }^{2,5}$ Meryle G Weinstein, 6,7,8,9,10 Robert C Cantu, 11,2Jordan Genece, 2,3,12,13,14Answorth A Allen, 2,3,15,16 Victor Lopez. 'University of Missouri, Missouri Orthopaedic Institute and Thompson Laboratory for Regenerative Orthopaedics, Columbia, USA; ${ }^{2}$ Rugby Research and Injury Prevention Group, Hospital for Special Surgery, New York, USA; ${ }^{3}$ Sports Performance Research Institute New Zealand, Auckland University of Technology, Auckland, New Zealand; ${ }^{4}$ New York University, College of Global Public Health, Urban Epidemiology Lab, New York, USA; ${ }^{5}$ New York University, Steinhardt School of Culture, Education and Human Development, New York, USA; ${ }^{6}$ Center for the Study of Traumatic Encephalopathy, Boston University School of Medicine, Boston, USA; ${ }^{7}$ Department of Neurosurgery and Sports Medicine, Emerson Hospital, Concord, USA; ${ }^{8}$ Sports Neurology and Concussion Program, Brigham and Women's Hospital, Boston, USA; ${ }^{9}$ Concussion Legacy Foundation, Waltham, USA: ${ }^{10}$ World Rugby, Independent Concussion Group, Dublin, Ireland; ${ }^{11}$ Mt. Sinai Hospital, Department of Dermatology, New York, USA; ${ }^{12}$ Sports Medicine and Shoulder Service, Hospital for Special Surgery, New York, USA; ${ }^{13}$ National Basketball Association, New York Knickerbockers, New York, USA; ${ }^{14}$ USA Basketball, Colorado Springs, USA; ${ }^{15}$ USA Rugby Empire and New England Geographic Union RFUs, New York/Boston, USA; ${ }^{16}$ Northeast Rugby Academy, USA Rugby Development Program and USOC-Community Olympic Development Program, New York/Boston, USA

\subsection{6/bjsports-2021-IOC.228}

Background Head and neck injuries are a collision sport concern, however there is a lack of Rugby-7s injury data, particularly in emerging Rugby nations.

Objective To determine the head, neck, and face (HNF) match injury rates and characteristics in US Rugby-7s.

Design Prospective descriptive epidemiology study.

Setting USA Rugby-sanctioned tournaments/series and championships (2010-2016). 